\title{
Optimization of laser-patterned YSZ-LSM composite cathode-electrolyte interfaces for solid oxide fuel cells
}

\author{
J.A. Cebollero ${ }^{\text {a }}$, M.A. Laguna-Bercero ${ }^{a}$, R. Lahoz ${ }^{b}$, J. Silva ${ }^{a}$, R. Moreno ${ }^{c}$, A. Larrea ${ }^{a}, *$ \\ ${ }^{a}$ Instituto de Ciencia de Materiales de Aragón, CSIC - U. Zaragoza, c/ María de Luna 3, E-50.018 Zaragoza, Spain

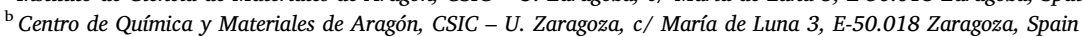 \\ ${ }^{\mathrm{c}}$ Instituto de Cerámica y Vidrio, CSIC, c/ Kelsen 5, Cantoblanco, E-28049 Madrid, Spain
}

\section{A R T I C L E I N F O}

\section{Keywords:}

SOFC

Laser-machining

Corrugated surface

Cathode polarization

Electrode-electrolyte interface

\begin{abstract}
A B S T R A C T
Patterned cathode/electrolyte interfaces formed by a hexagonal array of $\sim 22 \mu \mathrm{m}$ deep wells with $24 \mu \mathrm{m}$ lattice parameter have been prepared by pulsed laser machining to enlarge the contact surface and, consequently, to reduce the cathode polarization of Solid Oxide Fuel Cells. These new interfaces have been tested in YSZ-LSM/ YSZ/YSZ-LSM symmetrical cells, where the cathode is deposited by dip-coating. Appropriate ceramic suspensions have been formulated to penetrate into deep wells without presenting interfacial delamination after sintering. We analyse their applicability by comparing their rheology with the microstructure and electrochemical performance of the cells. The activation component of the polarization resistance is reduced by $\sim 50 \%$ using ethanol-based suspensions with $20 \mathrm{wt} \%$ solids loading, although the gas diffusion component increases due to excessive densification. Alternative ceramic suspensions with $17.5 \mathrm{wt} \%$ solids loading provide optimum electrode gas diffusion but lower activation components, resulting in an overall decrease of $\sim 20 \%$ in polarization resistance.
\end{abstract}

\section{Introduction}

Solid Oxide Fuel Cells (SOFCs) are clean and efficient energy conversion devices operating at high temperature (typically between $500^{\circ} \mathrm{C}$ and $1000^{\circ} \mathrm{C}$ ) [1]. They can also operate in reverse mode to produce pure hydrogen and oxygen from steam; the so-called Solid Oxide Electrolysis Cells (SOEC) [2]. Their principal characteristic is that they use a solid-phase oxide ion-conducting electrolyte. One of their main advantages is that, contrary to low temperature operation fuel cells, they are tolerant to $\mathrm{S}, \mathrm{CO}$ and $\mathrm{CO}_{2}$, as well as the fact that they can oxidize hydrocarbons [3].

In a SOFC electrode, the electrochemical reaction for fuel oxidation or oxygen reduction takes place at the triple phase boundary (TPB) regions, where the electronic phase, ionic phase, and porosity meet, ideally all of them percolating along the length of the electrode. In fact, a TPB is only active if an ion-conducting percolating network is formed up to the electrolyte. The volume fraction of components, tortuosity factor and TPB density are the key factors determining electrode performance. Based on recent studies and taking into account the different characteristic lengths, electrochemical reactions mostly take place in the vicinity of the electrode/electrolyte interface, typically within $10 \mu \mathrm{m}$ [4]. The microstructure of the electrodes is one of the main parameters governing cell power output and durability. Microstructure control in the porous electrode, within a length of about $1 \mu \mathrm{m}$ or less, is essential to achieve high power densities and low degradation rates. Traditionally the microstructure at the microscale range of the electrodes has been modified by controlling composition, grain size, pore formers, and sintering conditions. The electrode-electrolyte interface also has a relevant influence on the performance of the cell. Roughness and geometrical control at the mesoscale range $(10-30 \mu \mathrm{m})$, adherence between layers and interfacial surface are important parameters determining cell performance.

A few attempts exist in the literature to improve the electrolyte/ electrode interface performance by modifying its geometry at the mesoscale, although their results are still far from the implementation in SOFC fabrication. In fact, Wang et al. [5] modified this interface by the addition of convex YSZ (YSZ: $\mathrm{Zr}_{1-\mathrm{x}} \mathrm{Y}_{\mathrm{x}} \mathrm{O}_{2-\delta}$ ) particles by thermal spray on top of the electrolyte YSZ substrate. The convex structure was obtained by the deposition of surface-melted particles at the low particle temperature and velocity regime of the spray process. They found that the interfacial specific surface area increased by up to a factor of 3.45. Therefore cathode polarization of a cell with a modified cathode/ electrolyte interface is approximately one-third in comparison with a standard cell.

\footnotetext{
* Corresponding author.

E-mail address: alarrea@unizar.es (A. Larrea).
} 
Alternatively, Xu et al. [6] used a process where micropatterns were imprinted on the surface of a sheet of the ceramic material. They were able to obtain a dense ceramic sheet with different micropattern geometries (100 and $500 \mu \mathrm{m}$ of characteristic length) after debinding and sintering the ceramic sheets. They found that the finer pattern gives better performance, showing an increase by a factor of 3 in terms of power density, compared to a standard cell. Although the powder imprinting process is appropriate for complex-shaped components, scalability is an important issue as the imprinted area is relatively small. A similar approach was recently proposed by Farandos et al. [7]. They fabricated planar and 3D YSZ electrolyte structures by inkjet printing, where micro-pillar arrays and square lattices were subsequently printed resulting in crack-free 3D microstructures with a minimum feature resolution of $35 \mu \mathrm{m}$ in the horizontal plane.

Engraving the surface of the layer components is another alternative to increase the interface specific surface. According to Konno et al. [8], in order to have an improvement, the length of the structure should be greater (between 10 and $100 \mu \mathrm{m}$ ) than the effective active reaction zone of the electrode. They performed grooved structures by using the blasting method, showing that the machined areas do indeed contribute to the enhancement of the overall cell performance. The grooved cell performance was enhanced by $59 \%$ in power density with an interface enlargement factor of 1.73 . The groove width was $200 \mu \mathrm{m}$ at the surface and the distance between grooves was $250 \mu \mathrm{m}$, this being about the minimum width achievable using the blasting method. Another drawback of this method, apart from the limitation in groove density and being time consuming, is that the groove shape is not exactly reproducible. However, there is a significant improvement and the experimental evidence is confirmed by the calculations using theoretical models developed by different groups [9-12]. In fact, those predictions confirmed that not only is the polarization resistance reduced by corrugating the interfaces, as ohmic polarization could also be significantly improved, especially in electrode-supported cells with thin electrolytes. The total ohmic loss by ion transport and the mean activation overpotential in the mesoscale-corrugated cells decreased considerably due to the increase in the interfacial electrolyte/electrode contact surface. For example, according to recent numerical estimations from Kishimoto et al. [13] about mesoscale modification of thin electrolytes in anode supported SOFCs, the effect of periodic grooves of $20 \mu \mathrm{m}$ in depth, $30 \mu \mathrm{m}$ in width and $30 \mu \mathrm{m}$ apart is to produce a reduction of the ASR of about $30 \%$. From this reduction, $\sim 24 \%$ is on the polarization resistance and $\sim 35 \%$ corresponds to the ohmic contribution. Note that those estimations were performed for high current densities and they could differ from those near open circuit voltage (OCV) conditions. Thus, surface engraving opens a new research area for SOFC development and in fact it has attracted several groups in recent years [14-18].

In a very recent work, we have developed a different approach in order to enlarge the electrode-electrolyte interface in the mesoscale range (about 10 to $100 \mu \mathrm{m}$ ) by laser micro-patterning [17]. The beam of a $5 \mathrm{~ns}$ pulsed laser was used to engrave YSZ supports using a square array with a lattice parameter of $28 \mu \mathrm{m}$ and depths of about $7 \mu \mathrm{m}$, obtaining a decrease of the cathodic polarization resistance of about $30 \%$ with respect to non-processed cells. In this study we used conventional composite YSZ-LSM cathodes (LSM: $\mathrm{La}_{1-\mathrm{x}} \mathrm{Sr}_{\mathrm{x}} \mathrm{MnO}_{3}$ ). We focused our study on cathodic polarization because the oxygen reduction reaction kinetics on the cathode-electrolyte side is significantly slower than fuel reduction kinetics on the anode-electrolyte side, thus cathodic polarization is typically higher than anodic polarization [19]. This work demonstrated that the use of laser ablation to corrugate SOFC electrode/electrolyte interfaces is possibly the finest mechanising technology available from all the processing techniques previously mentioned. By this method, periodical surface designs not achievable by any other technique can be performed. In fact, there has been an increased interest in using laser machining for this purpose in recent years. Cai et al. [20] used laser micro-processing on both sides of electrolytesupported cells using lattice parameters of about $100 \mu \mathrm{m}$ and depths varying from 12 to $32 \mu \mathrm{m}$, followed by screen-printing deposition of standard electrodes (NiO/YSZ for the anode and LSM/YSZ for the cathode). They found that the structured interface extended the TPB length for electrochemical reaction, which led to an improvement in the power density of the modified cell of about $58 \%$ at $800{ }^{\circ} \mathrm{C}$. The same group performed similar experiments using anode supported cells [21]. Similar improvements, ranging from $47 \%$ to $55 \%$ in terms of power density, were also found at $800{ }^{\circ} \mathrm{C}$. Despite these improvements it is hard to make a proper comparison, as there can be significant differences in the thicknesses of all different layers. Finally, Gurauskis et al. [22] and Gu et al. [23] also used laser ablation to modify anode substrates. However, in both cases the main purpose of using this technique was to increase the porosity of the anode and thus, improve gas diffusion.

In order to exceed the positive results found in our previous work [17], the objective of this study is to further improve the performance of the cells by maximizing the contact surface area at the electrode/ electrolyte interface. For this purpose, hexagonal array patterns have been engraved by pulsed laser machining on YSZ plates and subsequently YSZ-LSM composite cathodes have been deposited on them. The composition and characteristics of different ceramic suspensions for dip coating deposition, as well as the electrochemical performance of the solid oxide cells formed, are analysed and discussed.

\section{Experimental procedure}

Commercial electrolyte substrates $8 \mathrm{YSZ}\left(8 \mathrm{~mol} \%\right.$ of $\mathrm{Y}_{2} \mathrm{O}_{3}$ ) were supplied by Kerafol $\mathrm{GmbH}$ (Germany) in the form of $50 \times 50 \mathrm{~mm}^{2}$ plates with a thickness of $150 \pm 15 \mu \mathrm{m}$. Micro-patterning of the ceramic substrates was performed using a Q-switched diode-pumped laser (Rofin PowerLine S3 SHG, Germany) emitting at a wavelength of $532 \mathrm{~nm}$. The nominal power is $2 \mathrm{~W}$ and the minimum pulse width $5 \mathrm{~ns}$. Additional details of the equipment used and the experimental set up can be found in references [17] and [24].

Surface characterization of the processed samples was performed using optical confocal profilometry (Sensofar PL $\mu 2300$, Spain). Microstructural analyses, in both plane view and in cross-section, were performed using field emission scanning electron microscopy (Carl Zeiss Merlin FESEM, Germany) equipped with Energy Dispersive Spectroscopy (EDS) (Oxford Instruments INCA-350 system, United Kingdom). Image analysis was performed using the Digital Micrograph software from Gatan Inc. (Pleasanton, CA, USA) on resin-infiltrated polished samples following the procedure described in reference [25]. The energy of the incident electrons for image analysis was set to $2 \mathrm{keV}$ to avoid electron penetration into the sample, mainly in the low density epoxy resin, and to get an accurate 2D representation of the sample's microstructure.

Symmetrical cells were fabricated using un-machined and patterned YSZ disks of $15 \mathrm{~mm}$ in diameter. LSM/YSZ oxygen electrodes were deposited on both sides by dip-coating with velocities of immersion and extraction of $4.5 \mathrm{~mm} / \mathrm{s}$. These electrodes were composed of a functional layer with composition LSM/YSZ 50/50 vol\% and a current collector layer of composition LSM/YSZ $80 / 20 \mathrm{vol} \%$, as described in reference [26]. The ceramic powders used were LSM20-P (surface area: $6 \pm 2$ $\mathrm{m}^{2} / \mathrm{g}$ and particle size $\mathrm{D}_{50}: 0.7-1.1 \mu \mathrm{m}$ ) from Fuel Cell Materials (USA) and TZ-8YS (surface area: $7 \pm 2 \mathrm{~m}^{2} / \mathrm{g}$ and particle size $\mathrm{D}_{50}: 0.09 \mu \mathrm{m}$, according to the supplier datasheet) from Tosoh (Japan). Sintering was performed using different loads on top to avoid deformation and to ensure appropriate electrolyte-electrode integration, as will be described in Section 3.2.

To get ceramic suspensions which can easily penetrate throughout the YSZ wells we needed to improve their wetting capacities. The two main factors to take into account for this purpose are the solids loading and the amount of binder. We selected ethanol and water as dispersing media. Ethanol is very convenient because of its fast drying, but water suspensions are able to accept higher solids loadings [27]. As dispersing 
agent (i.e., deflocculant) for the water-based suspensions, we used an ammonium salt of a poly(acrylic acid) (PAA) provided as Duramax D3005 (Rohm \& Haas, USA). The concentration of this component was selected from zeta potential experiments as a function of $\mathrm{pH}$ and as a function of PAA content, where the minimum amount of dispersant to get adequate negative zeta potential values was determined. Zeta potential measurements were performed using diluted suspensions $(0.1 \mathrm{~g}$ $1^{-1}$ ) with $\mathrm{KCl} 10^{-1} \mathrm{M}$ as inert electrolyte and adjusting the $\mathrm{pH}$ with $\mathrm{HCl}$ and $\mathrm{KOH}$. Measurements were performed with a NanoZS analyser (Malvern, UK). For the ethanol-based suspensions, we used a phosphate ester (Beycostat C213, CECA, France) as deflocculant and determined the appropriate amount by analysing the viscosity curves. We checked that these curves did not show significant thixotropy for a dispersant concentration higher than $0.5 \mathrm{wt} \%$, thus we selected this value for the slurry formulation. To achieve the required consistency for handling, Duramax B1000 (Rohm \& Haas, USA) and poly(vinyl butyral) (PVB, Eastman, USA) were selected as binder for the water-based suspensions and for the ethanol-based ones, respectively. The amount of binder was varied to get the desired viscosity. The idea was to change the solids loading and the amount of binder to obtain different suspensions in order to check their ability to penetrate into the laser-machined wells. The viscosity of the suspensions was determined using a Mars II rheometer from Thermo Haake (Germany). Measurements were performed using a double-cone and plate system with a diameter of $60 \mathrm{~mm}$ and a cone angle of $2^{\circ}$ at a temperature of $25^{\circ} \mathrm{C}$. Flow curves were obtained with a measuring cycle involving an increase of shear rate from 0 to $1000 \mathrm{~s}^{-1}$ for $5 \mathrm{~min}, 1 \mathrm{~min}$ dwell and a return to 0 shear rate for $5 \mathrm{~min}$.

Electrochemical Impedance Spectroscopy (EIS) was performed on symmetrical cells using a button-cell test rig (NorECs Probostat, Norway) and the data was collected using an electrochemical workstation (Zahner-Elektrik GmbH Zennium, Germany). EIS experiments were performed at temperatures ranging from $900{ }^{\circ} \mathrm{C}$ down to $750{ }^{\circ} \mathrm{C}$, at $50^{\circ} \mathrm{C}$ intervals, using a sinusoidal signal amplitude of $20 \mathrm{mV}$ and varying the frequency range from $100 \mathrm{kHz}$ to $100 \mathrm{mHz}$.

\section{Results and discussion}

\subsection{Preparation of patterned electrolyte-cathode surfaces and estimation of the interfacial area and volume}

In our previous publication [17] we laser-machined a square lattice of $\sim 7 \mu \mathrm{m}$ depth wells with lattice parameter $a=28 \mu \mathrm{m}$ on the surface of YSZ plates. For this surface we estimated, using optical profilometry, an enlargement of the electrode-electrolyte surface of about $20 \%$. In the present work we decided to go beyond these values preparing new surfaces with deeper profiles to reduce the cathodic polarization even further. We tested different patterns to get an arrangement of independent wells with depths in the range of $10-25 \mu \mathrm{m}$ and the minimum possible separation for this case. In this process we have to bear in mind not only the spot size at focus position, about $7 \mu \mathrm{m}$ in this case, but also the laser interaction with the substrate, the heat diffusion and the accuracy, repeatability and reproducibility of the beam positioning over the YSZ surface. Thus, this optimization was done in an empirical way. We determined that, for our experimental set-up, arrangements of wells with lattice parameter at least $24 \mu \mathrm{m}$ yielded to depths that increased linearly with the number of superimposed pulses. In this way, we machined different types of patterns with wells of $\sim 22 \mu \mathrm{m}$ in depth. In order to estimate the true surface area of the patterned electrolyte we decided not to use optical profilometry, as the optical characterization of transparent surfaces usually introduces a significant amount of noise. To suppress this noise, we were obliged to use Gaussian filters and the final result is highly dependent on the filter width. Hence, we calculated the surface area of single wells using their profile, digitalized from SEM cross-sections as shown in Fig. 1. Assuming that the cross-section represents a surface of revolution around the axis perpendicular to the plate surface and passing through the minimum $\mathrm{X}_{0}$, the surface area can be calculated using the following expressions:

$S^{s w}=2 \sum_{i} \pi r_{i} l_{i}$

where

$r_{i}=X_{0}-\frac{X_{i+1}-X_{i}}{2}$

and

$l_{i}=\sqrt{\left(X_{i+1}-X_{i}\right)^{2}+\left(Y_{i+1}-Y_{i}\right)^{2}}$

The enlargement factor, $\left(\mathrm{S} / \mathrm{S}_{0}\right)^{\mathrm{sw}}$, for a single well, calculated by the ratio of the well surface over its bearing surface, is about 2.4. Then, we have to take into account the packing factor. For a square arrangement of wells, $\left(\mathrm{S} / \mathrm{S}_{0}\right)^{\mathrm{Sq}}$, a hexagonal arrangement, $\left(\mathrm{S} / \mathrm{S}_{0}\right)^{\mathrm{Hex}}$, and a set of parallel grooves, $\left(\mathrm{S} / \mathrm{S}_{0}\right)^{\mathrm{Gr}}$, all of them with the lattice parameter equal to the width of the well $(a=24 \mu \mathrm{m})$, the enlargement factors give the following results:

$\left(\frac{S}{S_{0}}\right)^{S q}=\frac{\pi}{4}\left(\frac{S}{S_{0}}\right)^{s w}+(1-\pi / 4)$

$\left(\frac{S}{S_{0}}\right)^{H e x}=\frac{\pi}{2 \sqrt{3}}\left(\frac{S}{S_{0}}\right)^{s w}+\frac{\sqrt{3}-\pi / 2}{\sqrt{3}}$

$\left(\frac{S}{S_{0}}\right)^{G r}=\frac{\sum_{i} l_{i}}{S_{0}}$

The different enlargement factors calculated using the above expressions, and averaged for various experimental profiles, are collected in Table 1. As expected, the hexagonal arrangement offers the best results, because it corresponds to the closest-packed 2D disposition. The decrease with respect to a single well is only $-5 \%$, due to the small unprocessed zones between the wells that can be observed in Fig. 2. From the point of view of laser processing, to engrave a hexagonal arrangement has the same degree of complexity as the square arrangements or a set of grooves. Thus, the hexagonal arrangement was selected for this study. Although the increase in the electrolyte-electrode contact surface we can reach is high, about $130 \%$, we think that is not reasonable to expect a decrease in the polarization resistance of the same magnitude. We have to bear in mind that, when using a two phase composite cathode formed by a mixture of electronic and ionic conductors, the zone where the TPB points are located, and thus where oxygen reduction reactions (ORR) take place, is a volume near the surface, not just a surface. According to the estimations of Kishimoto et al. [4] using random-walk diffusion simulation of FIB-SEM anodes and 1D numerical simulations, the thickness of the active regions is about $10-15 \mu \mathrm{m}$, which is of the same order of magnitude as the depth and lattice parameter of the patterned surfaces. As the ORR kinetics in the cathode is slower than the $\mathrm{H}_{2}$ reduction kinetics in the anode, this value could probably be a bit lower, but the electrochemical active zone cannot yet be considered as a thin skin layer over the electrolyte. Hence, the expected increase in exchange current density due to the enlargement of the interfacial contact surface would not in fact be in correlation to the increase in contact surface, but to the increase in the volume of the region adjacent to the electrolyte $\left(V_{a c t}\right)$ compared with the volume of the same region in a flat, unprocessed region $\left(V_{o}\right)$. We have calculated this factor for the interfacial profile of Fig. 1 considering different thicknesses of the active region $(5-15 \mu \mathrm{m})$. The different regions are represented in Fig. 3. We determined that the increase in exchange current density could be between $40-80 \%$, which is still a significant figure and we think that it could be a reasonable estimation of the polarization decrease that is possible to reach with the pattern of Fig. 2. 


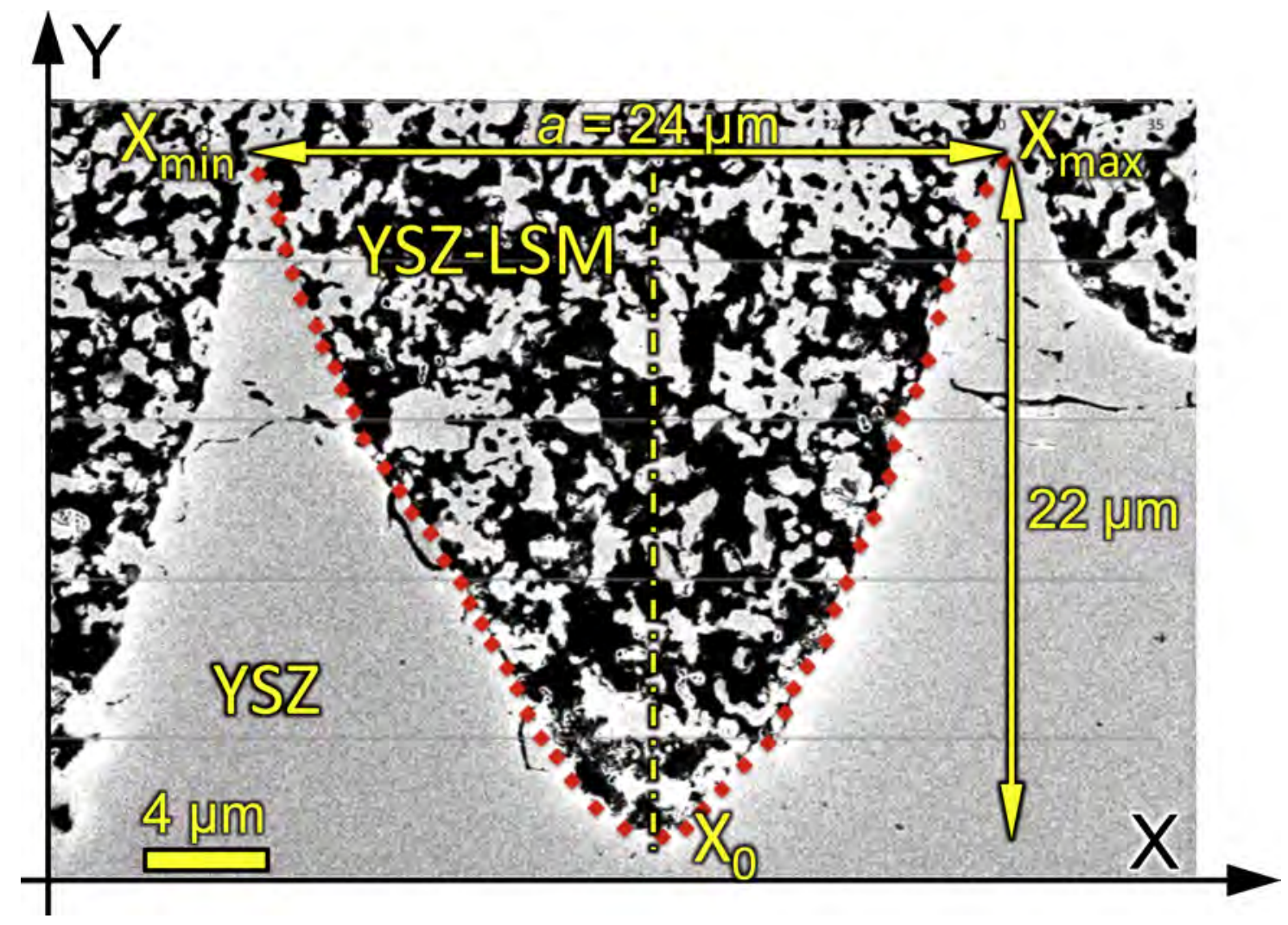

Fig. 1. Profile of a $22 \mu \mathrm{m}$ depth laser-machined single well digitized from its cross-section SEM image.

Table 1

Enlargement factors averaged for various profiles, as represented in Fig. 1, calculated for different arrangements of wells: single well, square lattice, hexagonal lattice and set of parallel grooves. In all cases the lattice parameter is $a=24 \mu \mathrm{m}$.

\begin{tabular}{llll}
\hline$\left(\mathrm{S} / \mathrm{S}_{0}\right)^{\mathrm{sw}}$ & $\left(\mathrm{S} / \mathrm{S}_{0}\right)^{\mathrm{Sq}}$ & $\left(\mathrm{S} / \mathrm{S}_{0}\right)^{\mathrm{Hex}}$ & $\left(\mathrm{S} / \mathrm{S}_{0}\right)^{\mathrm{Gr}}$ \\
\hline 2.44 & 2.13 & 2.30 & 2.18 \\
\hline
\end{tabular}

\subsection{Dip coating cathode formulations with high solids loadings}

In our previous study we deposited the cathodes by the dip-coating procedure using the same ceramic precursor that we used for flat unprocessed YSZ surfaces with good results in terms of overall microstructure and porosity [26]. The cathode was composed of two layers: an internal functional layer with 50-50 vol.\% LSM-YSZ and a current collector outer layer with $80-20$ vol.\% LSM-YSZ. The ceramic powders were dispersed in ethanol to a solids loading of $12.5 \mathrm{vol} \%$. The details about deflocculant and binder are included in Table 2, where this suspension is labelled SO (Suspension 0). Using this formulation, we

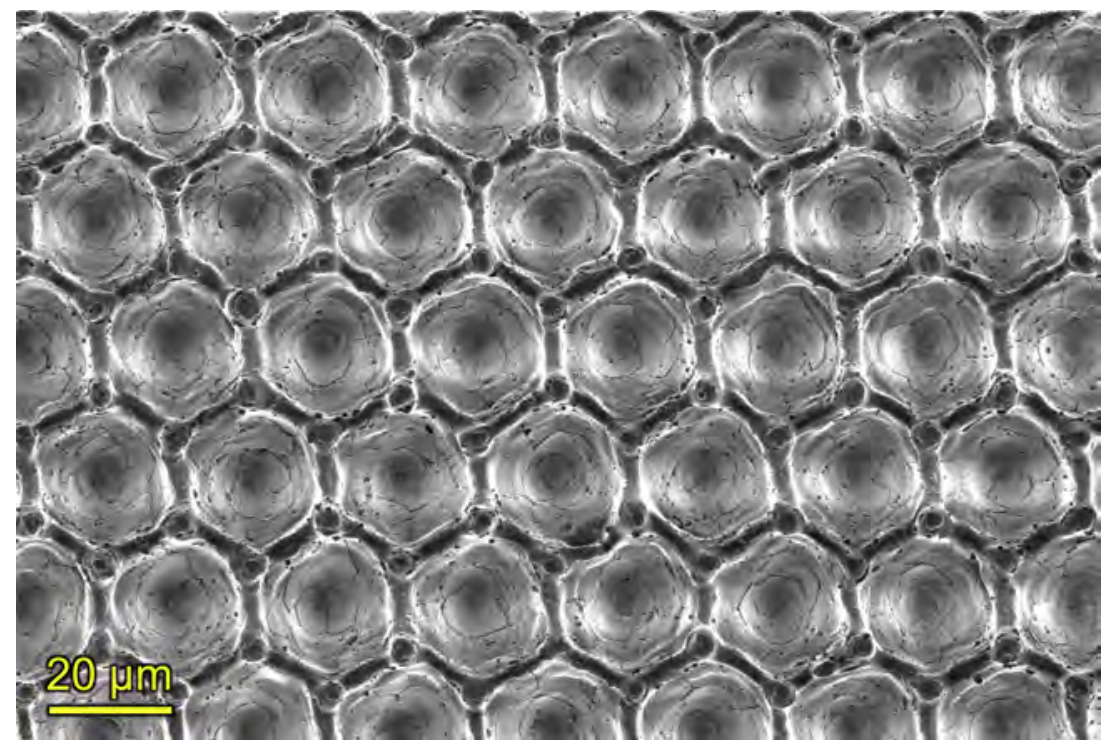

Fig. 2. Hexagonal arrangement of $22 \mu \mathrm{m}$ depth laser-machined wells on a YSZ plate (lattice parameter $a=24 \mu \mathrm{m}$ ). 


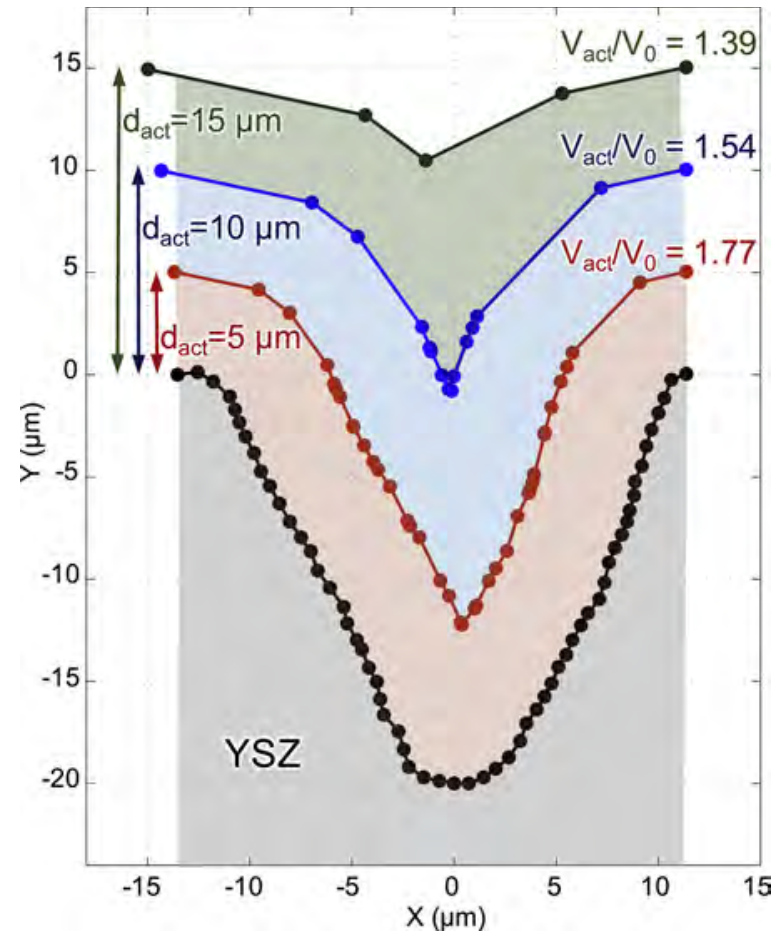

Fig. 3. Active volumes for the YSZ profile represented in Fig. 1 for 3 different active thicknesses $\left(d_{a c t}=5,10\right.$ and $\left.15 \mu \mathrm{m}\right)$. The cathodic active volume for $d_{a c t}=5 \mu \mathrm{m}$ is indicated in red; for $d_{a c t}=10 \mu \mathrm{m}$ it includes the red and the blue regions; for $d_{a c t}=15 \mu \mathrm{m}$ it includes the red, blue and green regions. $V_{O}$ is the volume of a cylinder of $24 \mu \mathrm{m}$ in diameter (the bearing area of the well) and height $d_{a c t}$ (For interpretation of the references to colour in this figure legend, the reader is referred to the web version of this article).

successfully averted the electrode-electrolyte debinding problems during sintering of the corrugated electrodes using an overpressure of $4.9 \mathrm{kPa}$ placing a load on top of the sample [17]. However, this approach, which worked very well for low-depth wells $(\sim 7 \mu \mathrm{m})$, is insufficient for deeper profiles $(\sim 22 \mu \mathrm{m})$. Even in the green state, the cathode-electrolyte adherence is poor due to inappropriate wetting, as can be observed in Fig. 4. For this reason, we decided to reformulate the ceramic suspension taking all the relevant aspects into consideration: solids loading, dispersing media and agent, as well as possible additives.

To characterise the new aqueous suspensions, the zeta potential of the starting powders was measured as a function of both the $\mathrm{pH}$ and the polyelectrolyte content. The zeta potential versus $\mathrm{pH}$ (not shown for sake of simplicity) showed that the isoelectric point of LSM was about 8, thus requiring high $\mathrm{pHs}$ to readily disperse that powder. In the case of YSZ the isoelectric point is about 2.3 [28]. Thus, to avoid heterocoagulation the use of an adsorbing polyelectrolyte would be required. In this case suspensions were prepared with PAA in order to shift down the isoelectric points as to allow working at neutral $\mathrm{pH}$. The evolution
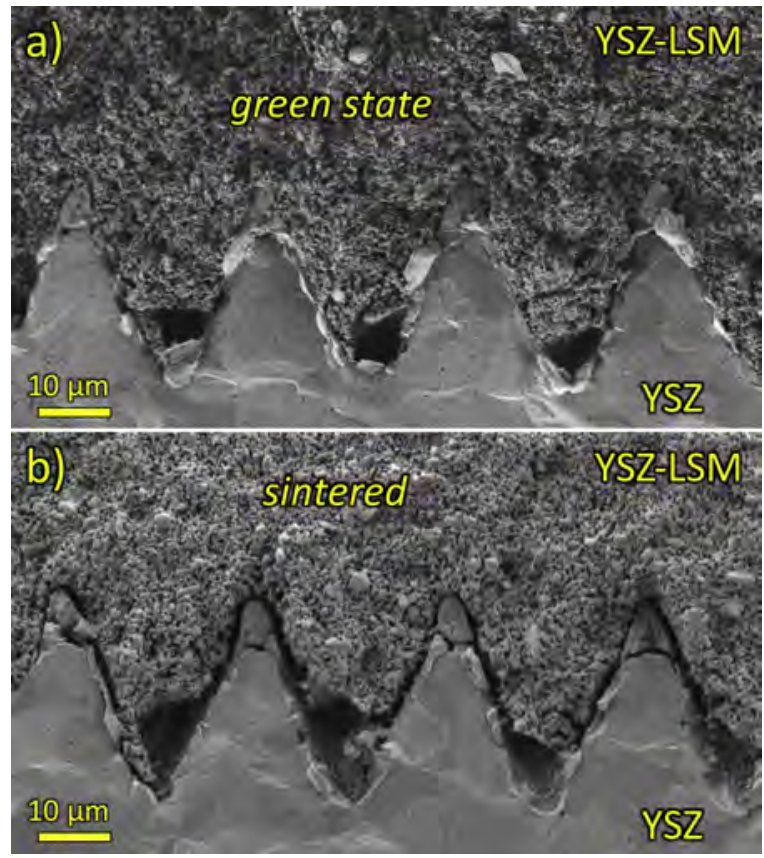

Fig. 4. Cathode-electrolyte interface in the green and sintered states. So formulation of the ceramic mixture was used for the dip-coating procedure. Cathode sintered for $2 \mathrm{~h}$ at $1150{ }^{\circ} \mathrm{C}$ with $19.6 \mathrm{kPa}\left(200 \mathrm{~g} / \mathrm{cm}^{2}\right)$ of overpressure.

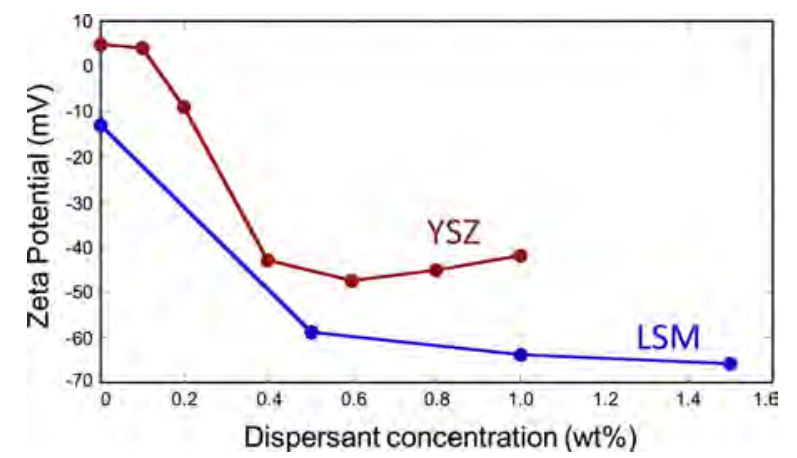

Fig. 5. Zeta potential as a function of the dispersant agent (Duramax D3005) content for LSM and YSZ powders in aqueous suspension.

of zeta potential with polyelectrolyte content is shown in Fig. 5. As it can be seen, both powders experiment a large increase of the absolute value of zeta potential with addition of PAA with maximum values above $45 \mathrm{mV}$ for $0.5 \mathrm{wt} \%$ deflocculant content. Thus, this was the concentration used for the preparation of the suspensions in water.

The suspension formulations and their main characteristics are collected in Table 2. $S 1$ and $S 2$ have higher solids loading than $S O$, but lower viscosities. In $S 3$ and $S 4$ we further increased the solids loading

Table 2

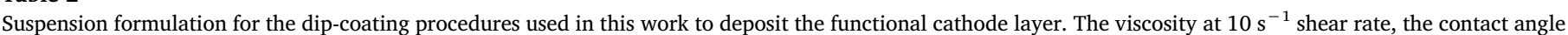

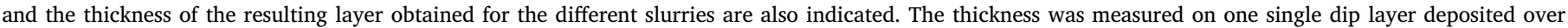
unprocessed YSZ plates. (BC213: Beycostat C213; D3005: Duramax D3005; DB1: Duramax B1000).

\begin{tabular}{|c|c|c|c|c|c|c|c|}
\hline Slurry & Dispersing medium & Solids Loading (vol\%) & Deflocculant (wt $\%$ ) & Binder (wt $\%)$ & Viscosity at $10 \mathrm{~s}^{-1}$ (mPa.s) & Contact angle $\left({ }^{\circ}\right)$ & Thickness $(\mu \mathrm{m})$ \\
\hline so & Ethanol & 12.5 & $1 \mathrm{BC} 213$ & 5 PVB & 72 & 66 & 15 \\
\hline$S 1$ & Ethanol & 20 & $0.5 \mathrm{BC} 213$ & 1.5 PVB & 59 & 60 & 20 \\
\hline$S 2$ & Ethanol & 20 & 0.5 BC213 & 0 PVB & 21 & 41 & 6 \\
\hline S3 & Water & 30 & 0.4 D3005 & 3 DB1 & 209 & 68 & 40 \\
\hline S4 & Water & 40 & 0.4 D3005 & 0 DB1 & 1058 & 72 & 130 \\
\hline S5 & Ethanol & 16 & $1 \mathrm{BC} 213$ & $5 \mathrm{PVB}$ & 523 & - & 19 \\
\hline$S 6$ & Ethanol & 17.5 & $1 \mathrm{BC} 213$ & $1 \mathrm{PVB}$ & 185 & - & 5 \\
\hline
\end{tabular}



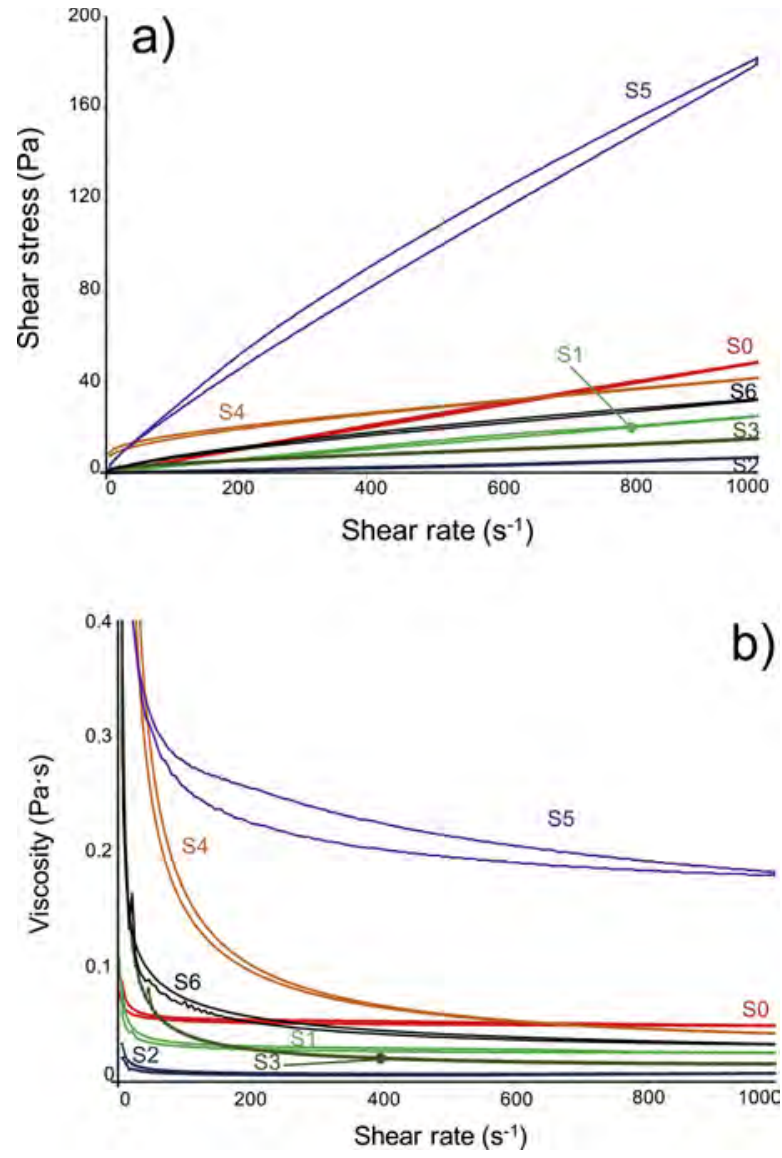

Fig. 6. Shear stress (a) and viscosity (b) as a function of the shear rate of the precursor slurries for dip-coating described in Table 2.

using water as dispersing medium. Again we varied the binder concentration to obtain different viscosities.

The flow and viscosity curves are presented in Fig. 6. The ethanolbased suspensions show Newtonian behaviour for shear rates higher that about $50 \mathrm{~s}^{-1}$, presenting viscosity values at low shear rates $10 \mathrm{~s}^{-1}$, which can be considered representative value for dip coating processes, of about 59 and $21 \mathrm{mPa} \cdot \mathrm{s}$ in the case of $S 1$ and $S 2$, respectively. However, the viscosity of the water-based slurries decreases with the shear rate, being significantly higher at low shear rates than that of ethanolbased slurries. Even in the case of $S 4$, where we did not include binder, its viscosity is higher because of its higher solids loading. Due to this behaviour, the static contact angle of the water-based suspensions is higher, resulting in significantly thicker layers. The thicknesses collected in Table 2 were measured on one single dip-coating layer deposited over unprocessed YSZ plates. The thickness of the cathodes obtained by dip-coating over patterned substrates is significantly higher. In Fig. 7, the SEM cross-sections of the resulting cathode layers deposited over patterned YSZ substrates are displayed. In all cases good electrode-electrolyte adhesion was obtained. However, we decided to study only the electrochemical behaviour of the electrodes prepared with ethanol as dispersing medium ( $S 1$ and $S 2$ ). The thickness of the layers obtained with water-based suspensions was excessive due to their high viscosity. In addition, ethanol-based slurries are more appropriate for our needs because of their higher drying speed and the homogeneity of the deposited layer thickness.

To check the electrochemical performance of the cathodes, symmetrical button cells with patterned electrolytes were prepared by dipcoating and subsequent sintering for $2 \mathrm{~h}$ at $1150^{\circ} \mathrm{C}$ with an overpressure of $19.6 \mathrm{kPa}\left(200 \mathrm{~g} / \mathrm{cm}^{2}\right)$. The polarization resistance values, comparing the results obtained in patterned electrodes and in unprocessed substrates, are collected in Table 3, whereas the corresponding Nyquist diagrams are displayed in Fig. 8. Ohmic contribution was subtracted from Nyquist diagrams for clarification purposes. As far polarization resistance, the patterned electrodes show lower values at low temperatures, $750{ }^{\circ} \mathrm{C}$, but not above $800^{\circ} \mathrm{C}$. Moreover, the average decrease in polarization resistance is much lower than expected and does not seem to correlate to the apparently good cathode-electrolyte contact. This result could be explained by fitting the impedance data to an equivalent model $L-R_{O^{-}}(R Q)_{1^{-}}(R Q)_{2^{-}}(R Q)_{3}-W_{4} . L$ is an inductance, $R_{i}$ are resistivity elements, $Q_{i}$ constant phase elements and $W_{4}$ a Warburg diffusion element. The values of the different fitting components are represented in Fig. 9. From these values it is evident that, using the new cathode formulation, a reduction of the $R_{2}$ and $R_{3}$ components is obtained. These two terms are associated with catalytic processes and are assigned to ionic transport in the YSZ matrix from the TPB to the electrode/electrolyte interface, as well as to the coupled processes of dissociative adsorption of oxygen and surface diffusion of oxygen species, respectively [29-31]. The reduction of both components represents the expected improvement in polarization resistance due to the enlargement of the electrode-electrolyte interfacial area, in concordance with previous results [17]. The $R_{1}$ component, usually attributed to the presence of secondary phases [32], has similar values in the patterned and unprocessed cathodes. However, a deterioration in the gas diffusion processes, indicated by the reduction of the resistivity term $\left(R_{4}\right)$ of the resistance from the Warburg element $\left(W_{4}\right)$, is also evident. In other words, the patterned cells seem to have a larger active reaction zone, as would be expected, but the gas transport has worsened. The overall cathode polarization only improves with the interfacial patterning at low temperature, but not at higher temperatures. The reason for this effect is that the gas diffusion component is mainly temperature independent, whereas the other processes are activated with the temperature, which explains the results of Table 3.

The origin of the increase of the gas diffusion component can be found in the microstructural analysis of the cathode. Image analysis of the cathodes on polished samples show that the cathode porosity, estimated as $32 \pm 1$ vol\% for $S 1$ and $28 \pm 1$ vol\% for $S 2$ are significantly lower than the reference value, $41 \pm 1 \mathrm{vol} \%$, obtained with $S O$ in unprocessed interfaces. Apparently, the significant increase in solids loading of the dip-coating precursor suspension, formulated to get good interfacial adhesion, also produced excessive densification of the electrode. The reduction in the amount of binder, which decomposes during sintering, might also slightly contribute to the porosity reduction. Hence, gas diffusion is hampered by these low porosity values, below the percolation threshold. This effect is more important in the bottom of the wells, where, in addition, the lateral contribution to gas diffusion is very low. To get a better picture of how these new cathode formulations work on depth patterned profiles, we have also represented in Table 3 the polarization resistance after subtraction of the gas diffusion component $\left(R_{4}\right)$. The best results are obtained for $S 2$ formulation, where polarization resistance decreased from values of $25 \%$ at $750{ }^{\circ} \mathrm{C}$ to $50 \%$ at $900{ }^{\circ} \mathrm{C}$. On average, the polarization decrease for the whole temperature range is about $35 \%$, which is more or less in agreement with the estimations given in section 3.1. There, we anticipated an increase in the electrochemical active volume of between $\sim 40 \%$ to $80 \%$, depending on the thickness of the active area close to the cathode-electrolyte interface $(5-15 \mu \mathrm{m})$.

\subsection{Dip coating cathode formulations with intermediate solids loadings}

The results of Section 3.2 for the activation polarization decrease are encouraging. However, we have to restore the proper cathode porosity to also ensure low concentration polarization. Thus, we decided to explore two new suspensions, labelled as $S 5$ and $S 6$ in Table 2, selecting intermediate solids loadings between $S O$ and $S 2$. In turn, we reduced the amount of binder in $S 6$, trying to compensate the influence of the higher solids loading in the cathode porosity. The 

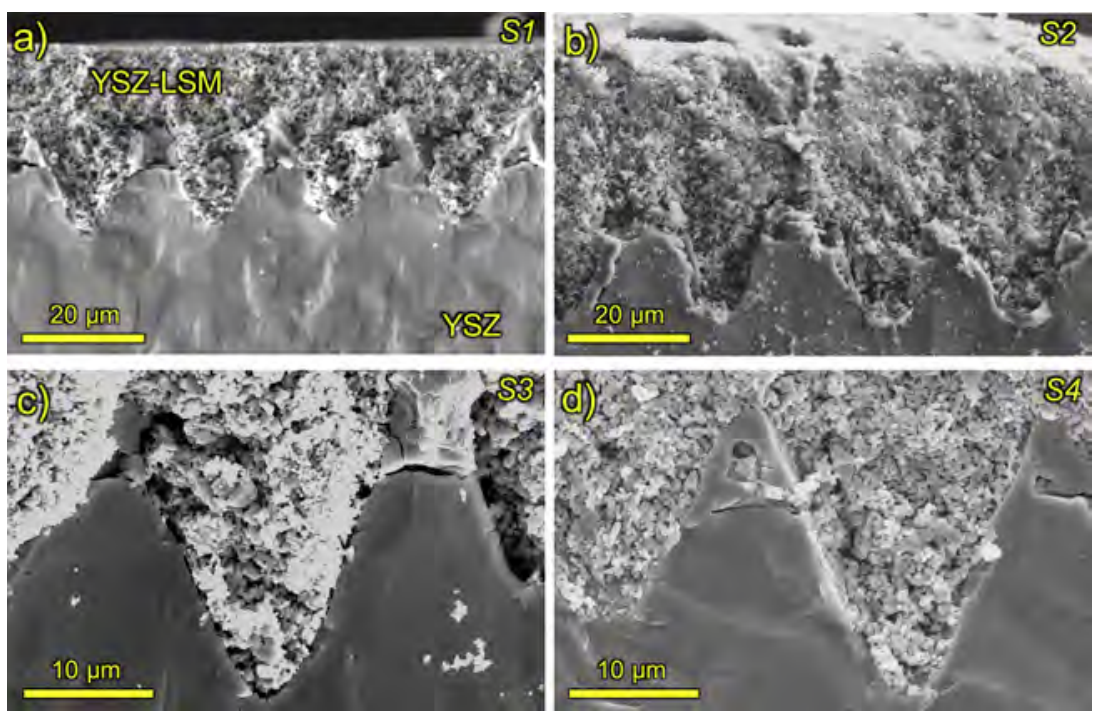

Fig. 7. SEM cross-section images of LSM-YSZ cathodes deposited through multistep dipping with 4 consecutive immersions of patterned YSZ plates and then sintered. Images (a)-(d) correspond to suspensions S1-S4, respectively.

Table 3

Polarization resistance $\left(\Omega \cdot \mathrm{cm}^{2}\right)$ of symmetrical cells formed with unprocessed and patterned electrode-electrolyte interfaces using cathodes prepared with suspensions $S 1$ and $S 2$. For each case the full value $\left(R_{p o l}\right)$ and the value after subtraction of the $R_{4}$ component $\left(R_{a c t}=R_{p o l}-R_{4}\right)$, attributed to gas diffusion, is indicated.

\begin{tabular}{llllll}
\hline $\begin{array}{l}\mathrm{T} \\
\left({ }^{\circ} \mathrm{C}\right)\end{array}$ & $\begin{array}{l}S 1 \\
\text { unprocessed }\end{array}$ & $\begin{array}{l}S 1 \\
\text { patterned }\end{array}$ & $\begin{array}{l}-\Delta \mathrm{R} \\
(\%)\end{array}$ & $\begin{array}{l}S 2 \\
\text { unprocessed }\end{array}$ & $\begin{array}{l}S 2 \\
\text { patterned }\end{array}$ \\
\hline & $R_{\text {pol }}-R_{\text {act }}$ & $R_{\text {pol }}-R_{\text {act }}$ & $R_{\text {pol }}-R_{\text {act }}$ & $R_{\text {pol }}-R_{\text {act }}$ & $\begin{array}{l}-\Delta \mathrm{R} \\
(\%)\end{array}$ \\
750 & $0.77-0.77$ & $0.72-0.72$ & $6.5-6.5$ & $0.80-0.73$ & $R_{\text {pol }}-R_{\text {act }}$ \\
800 & $0.39-0.37$ & $0.37-0.33$ & $5.1-11$ & $0.45-0.35$ & $0.75-0.55$ \\
850 & $0.21-0.16$ & $0.19-0.13$ & $9.5-19$ & $0.24-0.15$ & $0.40-0.21$ \\
900 & $0.13-0.07$ & $0.12-0.06$ & $7.7-14$ & $0.17-0.08$ & $0.25-0.09$ \\
& & & & $-4.18-0.04$ \\
\hline
\end{tabular}

viscosity curves of these suspensions are also included in Fig. 6. S5 presents high viscosity values compared to the other ethanol-based formulations, but $S 6$ presents similar values to $S 1$. SEM cross-sections showing the cathode penetration inside the wells are shown in Fig. 10. We can observe that cathode-electrolyte adhesion for $S 5$ is defective, but it seems to be correct for $S 6$. Thus, we decided to further analyse only the cathodes prepared with $S 6$. The cathode porosity was studied by image analysis in SEM on resin-infiltrated polished cross-sections. The SEM images were masked using both previously published [33] and dedicated scripts written for Digital Micrograph (Gatan, CA USA) software, to discriminate the zones inside the wells and above them. In the areas above the wells we obtained porosity values of $40 \pm 1 \mathrm{vol} . \%$, as required. But in the inner zones inside the wells the porosity increased up to $50 \pm 1$ vol.\%. This could be due to high viscosity of $S 5$ that is not able to completely fill the cavity of the well. This is clearly softened in the case of $S 6$, with a viscosity nearly three times lower. It must be taken into account that, whereas in the case of tape casting higher binder contents are necessary to allow the tape removal, the reduction of binder content helps to improve the adherence in the present case.

The electrochemical performance of the patterned cells formed using $S 6$ as cathode formulation was again checked using symmetrical button cells. The sintering cycle was the same as for symmetrical cells fabricated with $S 1$ and $S 2$, but using lower overpressure, $4.9 \mathrm{kPa} \mathrm{(50 \textrm {g } /}$ $\mathrm{cm}^{2}$ ), to avoid any risk of excessive densification. The polarization resistance values (including the individual resistance components), comparing patterned and unprocessed substrates, are summarized in Table 4. The corresponding Nyquist diagrams are also displayed in Fig. 11. Ohmic contribution was subtracted from Nyquist diagrams for clarification purposes. Regarding this contribution, an improvement is also expected for the patterned samples, as the thickness of the electrolyte was slightly reduced (about $20 \%$ of its original $150 \mu \mathrm{m}$ thickness). In fact, an average reduction of about $26 \%$ for the range of measured temperatures was obtained. In addition, polarization resistance was also improved by about $16 \%$ on average. If we analyse in detail the different resistance values from equivalent circuit fitting, as listed in Table 4, it is important to point out that $R_{1}$ (attributed to the presence of secondary phases) disappeared when using these new formulations, possibly as a result of the lower overpressure used. In addition, $R_{2}$ and $R_{3}$ (assigned to activation polarization) were reduced for the patterned cell by about $20 \%$. As previously explained, the reduction of both components was expected, due to the enlargement of the electrode-electrolyte interfacial area. In any case, this reduction is lower than expected according to our estimations in Section 3.1 (enhancement expected between $40-80 \%$ ). The reason for this effect can be attributed to the increase of porosity inside the wells, as previously mentioned. If we observe the fitted values for $R_{4}$ (concentration polarization), there are no significant differences between patterned and unprocessed cells, as the diffusion limitation previously observed is now avoided due to the reformulation of the LSM-YSZ slurry. This result confirms that the microstructure of the LSM-YSZ using S6 is now optimized in terms of diffusion, but still requires further optimization in order to maximize catalytic activity.

\section{Conclusions}

Patterned cathode/electrolyte interfaces have been produced by pulsed laser micro-machining to reduce the cathode activation polarization of Solid Oxide Fuel Cells. The performance of these interfaces have been tested in YSZ symmetrical cells, where the YSZ-LSM composite cathode is deposited by dip-coating. As conventional ceramic suspensions commonly used for this purpose are not useful in this case, 

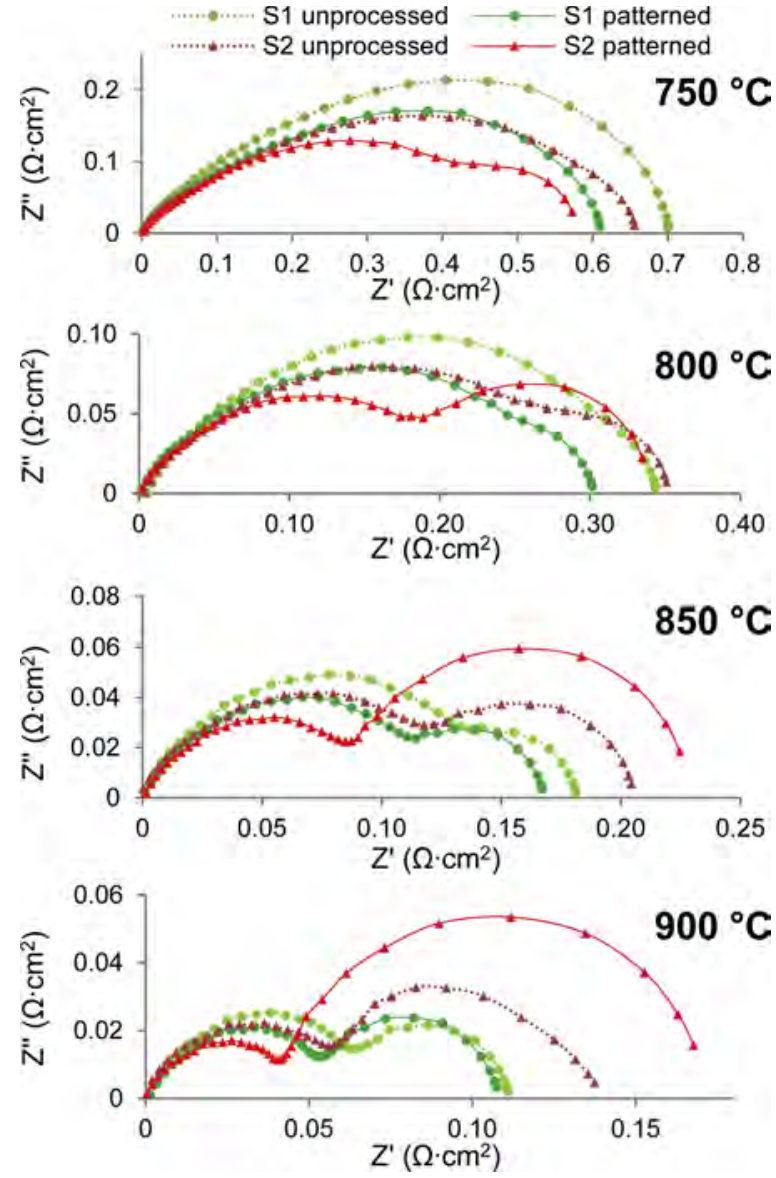

Fig. 8. Nyquist diagrams of the symmetrical cells formed with patterned cathode-electrolyte interfaces using $S 1$ and $S 2$ formulations of Table 2 and compared with the diagrams obtained with the same cathodes deposited on unprocessed YSZ surfaces. Ohmic contribution was subtracted from the diagrams for easier comparison.

we have prepared new ceramic formulations, with increasing solids loadings and appropriate viscosity, able to penetrate and sinter into deep laser-machined wells. The properties and applicability of the different suspensions have been analysed comparing the electrochemical performance of the cells with their rheological properties and the
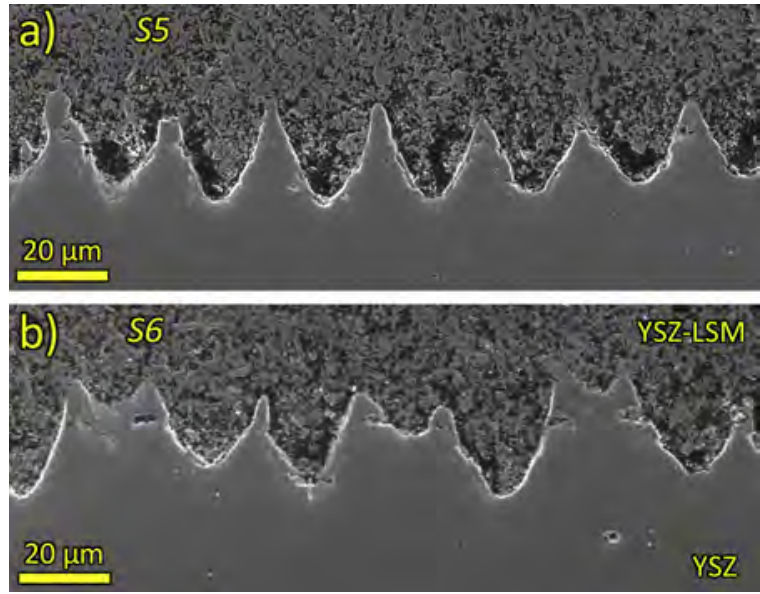

Fig. 10. SEM cross-section images of LSM-YSZ cathodes deposited over patterned YSZ plates and then sintered using formulations defined as $S 5$ and $S 6$ in Table 2. They correspond respectively to images (a) and (b).

Table 4

Summary of resistance values obtained from the fitting of the EIS experiments of Fig. 11 to an equivalent model $L-R_{O^{-}}(R Q)_{2}-(R Q)_{3}-W_{4}$.

\begin{tabular}{|c|c|c|c|c|c|c|c|c|}
\hline \multirow[t]{2}{*}{$\mathrm{T}\left({ }^{\circ} \mathrm{C}\right)$} & \multicolumn{4}{|c|}{ S6 unprocessed } & \multicolumn{4}{|c|}{$S 6$ patterned } \\
\hline & $R_{p o l}$ & $R_{2}$ & $R_{3}$ & $R_{4}$ & $R_{p o l}$ & $R_{2}$ & $R_{3}$ & $R_{4}$ \\
\hline 750 & 1.24 & 0.17 & 0.8 & 0.27 & 1.20 & 0.15 & 0.74 & 0.31 \\
\hline 800 & 0.59 & 0.14 & 0.34 & 0.11 & 0.49 & 0.11 & 0.28 & 0.10 \\
\hline 850 & 0.31 & 0.09 & 0.18 & 0.04 & 0.24 & 0.07 & 0.15 & 0.02 \\
\hline 900 & 0.18 & 0.04 & 0.11 & 0.03 & 0.14 & 0.06 & 0.02 & 0.06 \\
\hline
\end{tabular}

microstructure of the as-deposited cathodes. The best results for the reduction of the activation component of the polarization resistance are obtained using an ethanol-based formulation with $20 \mathrm{wt} \%$ solids loading and a viscosity of $21 \mathrm{mPa} \cdot \mathrm{s}$ at $10 \mathrm{~s}^{-1}$ of shear rate ( $S 2$ formulation). Under these conditions we obtained a reduction of $\sim 50 \%$, although the gas diffusion component increased significantly due to excessive cathode densification. Concentration polarization was thus reduced using a suspension formulation with $17.5 \mathrm{wt} \%$ solids loading and $185 \mathrm{mPa} \cdot \mathrm{s}$ viscosity at $10 \mathrm{~s}^{-1}$ shear rate ( $S 6$ formulation). With this formulation we restored the appropriate electrode gas diffusion, but the activation component decrease obtained in this manner, about $20 \%$, is
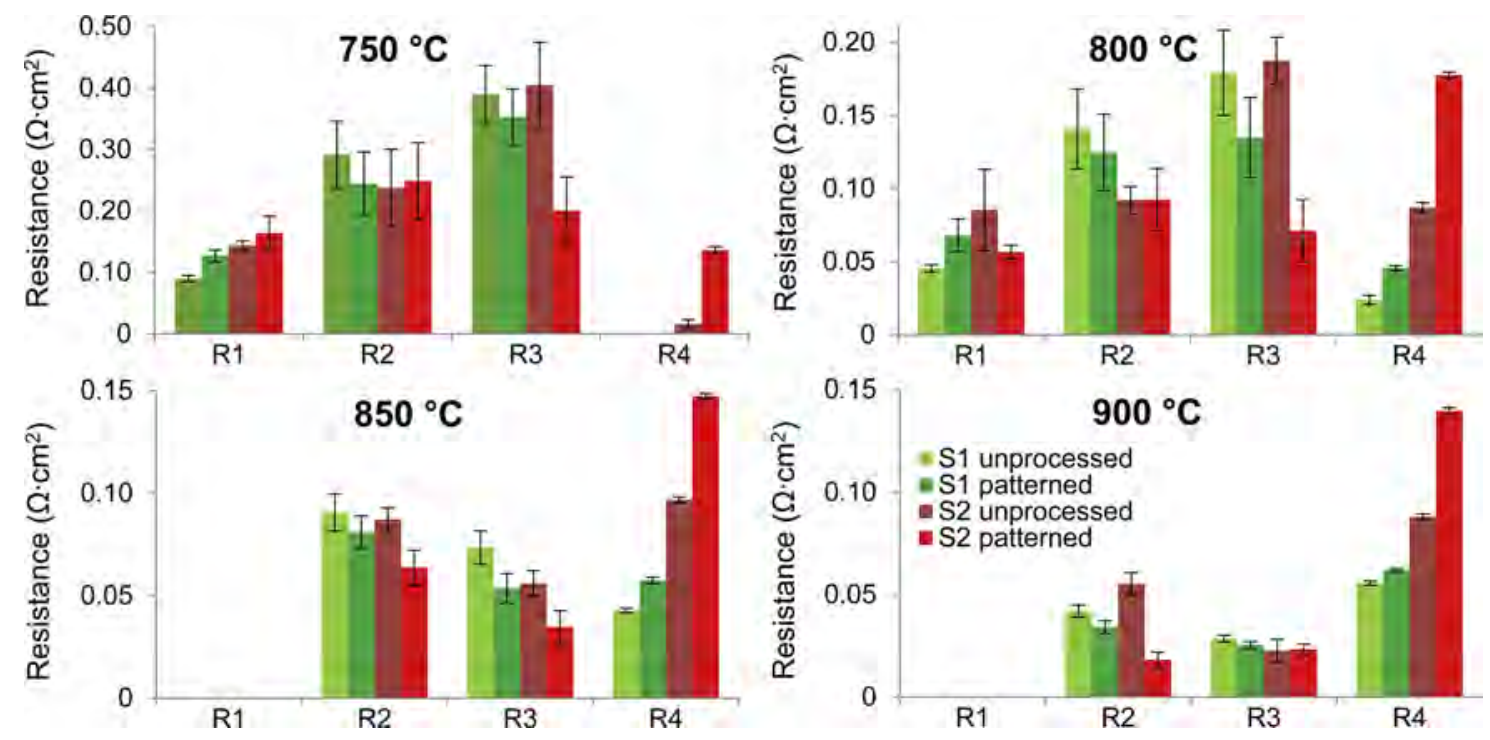

Fig. 9. Values resulting from the fitting of the EIS experiments of Fig. 8 to an equivalent model $L-R_{0^{-}}(R Q)_{1^{-}}(R Q)_{2}-(R Q)_{3}-W_{4}$. 


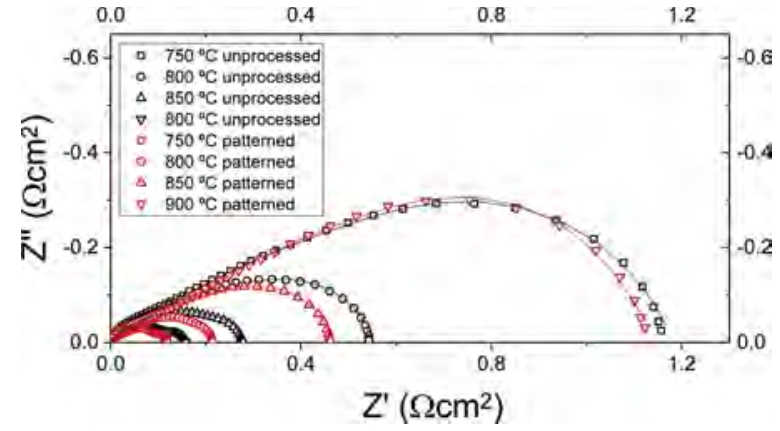

Fig. 11. Nyquist diagrams of the symmetrical cells fabricated with patterned cathode-electrolyte interfaces using $S 6$ formulation and compared with the diagrams obtained with the same cathodes deposited on unprocessed YSZ surfaces.

lower than expected, probably because of the excessive porosity of the cathode in the regions inside the wells. Further optimization is still needed to combine the best properties of $S 2$ and $S 6$ formulations to get the optimum ceramic suspension.

\section{Acknowledgments}

This work was funded by the MAT2015-68078-R and MAT201567586-C3-2-R projects, which are financed by the Spanish Government (Ministerio de Economía y Competitividad) and the Feder Program of the European Union. Fruitful discussion with H. Iwai and M. Kishimoto are kindly acknowledged.

\section{References}

[1] S.C. Singhal, K. Kendall, High Temperature Solid Oxide Fuel Cells: Fundamentals, Design and Applications, Elsevier, 2003.

[2] M.A. Laguna-Bercero, Recent advances in high temperature electrolysis using solid oxide fuel cells: a review, J. Power Sources 203 (2012) 4-16.

[3] T.M. Gur, Critical review of carbon conversion in "Carbon Fuel Cells", Chem. Rev. 113 (8) (2013) 6179-6206.

[4] M. Kishimoto, H. Iwai, M. Saito, H. Yoshida, Quantitative evaluation of solid oxide fuel cell porous anode microstructure based on focused ion beam and scanning electron microscope technique and prediction of anode overpotentials, J. Power Sources 196 (10) (2011) 4555-4563.

[5] X.-M. Wang, C.-X. Li, J.-Y. Huang, G.-J. Yang, C.-J. Li, Deposition mechanism of convex YSZ particles and effect of electrolyte/cathode interface structure on cathode performance of solid oxide fuel cell, Int. J. Hydrogen Energy 39 (25) (2014) $13650-13657$.

[6] Y. Xu, F. Tsumori, T. Osada, H. Miura, Improvement of solid oxide fuel cell by imprinted micropatterns on electrolyte, Micro Nano Lett. 8 (10) (2013) 571-574.

[7] N.M. Farandos, L. Kleiminger, T. Li, A. Hankin, G.H. Kelsall, Three-dimensional inkjet printed solid oxide electrochemical reactors. I. Yttria-stabilized Zirconia Electrolyte, Electrochim. Acta 213 (2016) 324-331.

[8] A. Konno, H. Iwai, M. Saito, H. Yoshida, A corrugated mesoscale structure on electrode-electrolyte interface for enhancing cell performance in anode-supported SOFC, J. Power Sources 196 (18) (2011) 7442-7449.

[9] A. Konno, H. Iwai, K. Inuyama, A. Kuroyanagi, M. Saito, H. Yoshida, K. Kodani, K. Yoshikata, Mesoscale-structure control at anode/electrolyte interface in solid oxide fuel cell, J. Power Sources 196 (1) (2011) 98-109.

[10] J.H. Nam, D.H. Jeon, A comprehensive micro-scale model for transport and reaction in intermediate temperature solid oxide fuel cells, Electrochim. Acta 51 (17) (2006) $3446-3460$.

[11] A. Chesnaud, F. Delloro, M. Geagea, A.P. Abellard, J. Ouyang, D. Li, T. Shi, B. Chi, R. Ihringer, M. Cassir, A. Thorel, Corrugated electrode/electrolyte interfaces in SOFC: theoretical and experimental development, in: S.C. Singhal, T. Kawada (Eds.), Solid Oxide Fuel Cells 15, Electrochemical Soc Inc, Pennington, 2017, pp. 1851-1863.

[12] F. Delloro, M. Viviani, Simulation study about the geometry of electrode-electrolyte contact in a SOFC, J. Electroceramics 29 (3) (2012) 216-224.

[13] M. Kishimoto, M. Sasaki, H. Iwai, H. Yoshida, Numerical assessment of mesoscale modification of thin electrolyte in anode-supported solid oxide fuel cells, 13th European SOFC \& SOE Forum (2018) 46-55.

[14] L. Bi, S.P. Shafi, E.H. Da'as, E. Traversa, Tailoring the cathode-electrolyte interface with nanoparticles for boosting the solid oxide fuel cell performance of chemically stable proton-conducting electrolytes, Small 14 (32) (2018).

[15] G.F. Cai, Y.H. Gu, L. Ge, Y.L. Zhang, H. Chen, L.C. Guo, Modification of electrolyte surface with "windows" and "dimples array" structure for SOFC based on YSZ electrolyte, Ceram. Int. 43 (12) (2017) 8944-8950.

[16] J.M. Xie, W.Q. Hao, F.H. Wang, Interface strength analysis of the corrugated anodeelectrolyte interface in solid oxide fuel cell characterized by peel force, J. Power Sources 396 (2018) 141-147.

[17] J.A. Cebollero, R. Lahoz, M.A. Laguna-Bercero, A. Larrea, Tailoring the electrodeelectrolyte interface of Solid Oxide Fuel Cells (SOFC) by laser micro-patterning to improve their electrochemical performance, J. Power Sources 360 (2017) 336-344.

[18] S. Masciandaro, M. Torrell, P. Leone, A. Tarancón, Three-dimensional printed yttria-stabilized zirconia self-supported electrolytes for solid oxide fuel cell applications, J. Eur. Ceram. Soc. (2018).

[19] S.B. Adler, Factors governing oxygen reduction in solid oxide fuel cell cathodes, Chem. Rev. 104 (10) (2004) 4791-4843.

[20] G.F. Cai, Y.L. Zhang, H.L. Dai, S.C. He, L. Ge, H. Chen, L.C. Guo, Modification of electrode/electrolyte interface by laser micro-processing for solid oxide fuel cell, Mater. Lett. 195 (2017) 232-235.

[21] Y.L. Zhang, G.F. Cai, Y.H. Gu, L. Ge, Y.F. Zheng, H. Chen, L.C. Guo, Modifying the electrode-electrolyte interface of anode supported solid oxide fuel cells (SOFCs) by laser-machining, Energy Convers. Manage. 171 (2018) 1030-1037.

[22] J. Gurauskis, D. Sola, J.I. Pena, V.M. Orera, Laser drilling of Ni-YSZ cermets, J. Eur. Ceram. Soc. 28 (14) (2008) 2673-2680.

[23] D.G. Gu, N. Shi, F. Yu, Y.F. Zheng, H. Chen, L.C. Guo, Asymmetric anode substrate fabricated by phase inversion process and its interface modification for solid oxide fuel cells, J. Alloys. Compd. 742 (2018) 20-28.

[24] J.A. Cebollero, R. Lahoz, M.A. Laguna-Bercero, J.T. Pena, A. Larrea, V.M. Orera, Characterization of laser-processed thin ceramic membranes for electrolyte-supported solid oxide fuel cells, Int. J. Hydrogen Energy 42 (19) (2017) 13939-13948.

[25] S. Serrano-Zabaleta, M.A. Laguna-Bercero, L. Ortega-San-Martin, A. Larrea, Orientation relationships and interfaces in directionally solidified eutectics for solid oxide fuel cell anodes, J. Eur. Ceram. Soc. 34 (9) (2014) 2123-2132.

[26] H. Monzon, M.A. Laguna-Bercero, A. Larrea, B.I. Arias, A. Varez, B. Levenfeld, Design of industrially scalable microtubular solid oxide fuel cells based on an extruded support, Int. J. Hydrogen Energy 39 (10) (2014) 5470-5476.

[27] V. Moreno, M. Bernardino, D. Hotza, Mechanical behavior of Yttria-stabilized zirconia aqueous cast tapes and laminates, J. Ceram. (2014) 713916.

[28] R. Fernandez-Gonzalez, T. Molina, S. Savvin, R. Moreno, A. Makradi, P. Nunez, Fabrication and electrical characterization of several YSZ tapes for SOFC applications, Ceram. Int. 40 (9) (2014) 14253-14259.

[29] M.J. Jorgensen, M. Mogensen, Impedance of solid oxide fuel cell LSM/YSZ composite cathodes, J. Electrochem. Soc. 148 (5) (2001) A433-A442.

[30] J.D. Kim, G.D. Kim, J.W. Moon, Y.I. Park, W.H. Lee, K. Kobayashi, M. Nagai, C.E. Kim, Characterization of LSM-YSZ composite electrode by ac impedance spectroscopy, Solid State Ion. 143 (3-4) (2001) 379-389.

[31] E.P. Murray, T. Tsai, S.A. Barnett, Oxygen transfer processes in (La,Sr)MnO3/Y2O3stabilized ZrO2 cathodes: an impedance spectroscopy study, Solid State Ion. 110 (3-4) (1998) 235-243.

[32] J. Nielsen, J. Hjelm, Impedance of SOFC electrodes: a review and a comprehensive case study on the impedance of LSM:YSZ cathodes, Electrochim. Acta 115 (2014) 31-45.

[33] D.R.G. Mitchell, B. Schaffer, Scripting-customised microscopy tools for Digital Micrograph(TM), Ultramicroscopy 103 (4) (2005) 319-332. 